\title{
Transcranial Doppler Monitoring During Carotid Endarterectomy:
}

\section{A Technical Case Report}

\author{
Eric J. Heyer ${ }^{*}{ }^{* \star}$, Christopher J. Winfree $^{\dagger}$, William J. Mack ${ }^{\dagger}$, and E. Sander Connolly Jr. ${ }^{\dagger}$ \\ ${ }^{*}$ Department of Anesthesiology, The New York Presbyterian Hospital, Columbia University College of \\ Physicians and Surgeons, New York, New York \\ ${ }^{* *}$ Department of Neurology, The New York Presbyterian Hospital, Columbia University College of Physicians \\ and Surgeons, New York, New York \\ $\dagger$ Department of Neurosurgery, The New York Presbyterian Hospital, Columbia University College of \\ Physicians and Surgeons, New York, New York
}

\section{Summary}

We report a case involving a patient undergoing carotid endarterectomy in whom transcranial doppler monitoring demonstrated impaired cerebral blood flow during the initial dissection of the carotid artery, during cross-clamping of the carotid artery, and after the shunt was kinked inadvertently. Only when the carotid artery was cross-clamped were these ischemic changes also seen by electroencephalography. During the other episodes, the electroencephalography tracings demonstrated no detectable changes. This case illustrates the importance of using multiple modalities to determine the adequacy of cerebral blood flow and neuronal integrity.

\section{Keywords}

Carotid endarterectomy; Surgery; Transcranial Doppler

\begin{abstract}
Transcranial Doppler ultrasonography (TCD) has been used for diagnostic purposes to determine the adequacy of cerebral blood flow (CBF) in the major cerebral vessels (1). More recently, TCD monitoring has been used to determine the adequacy of CBF following crossclamping during carotid endarterectomy (CEA) (2). Additionally, TCD may reveal the presence of cerebral emboli showers in the middle cerebral artery upon release of the carotid cross-clamp $(3,4)$. We report a case of a patient undergoing CEA in whom intraoperative TCD monitoring was used as an adjunct to electroencephalography (EEG) monitoring. The TCD monitor clearly indicated that flow in the carotid artery was impaired during the initial dissection of the carotid artery, cross-clamping of the carotid artery, manipulation and kinking of the shunt by the surgeon, and removal of the shunt. The EEG demonstrated detectable changes only during cross-clamping prior to insertion of a shunt and at removal of the shunt. Transcranial Doppler ultrasonography provides beat-to-beat monitoring of the integrity of the shunt before EEG changes are manifest, and in so doing provides real-time information for the surgeon, permitting immediate changes in operative technique.
\end{abstract}

Address correspondence and reprint requests to Eric J. Heyer, M.D., Ph.D., Departments of Anesthesiology and Neurology, Columbia University, PH 5-523, 630 West $168^{\text {th }}$ Street, New York, NY 10032-3784. 


\section{Case Report}

Patient History

The patient was a 61-year-old man with a past medical history significant for hypertension, hypercholesterolemia, and a cerebrovascular accident involving the right cerebellum eight months before admission. A cerebral angiogram at that time revealed right carotid artery occlusion and high-grade stenosis of the left carotid artery (> 70\%). He was started on both antihypertensive therapy and anticoagulation with warfarin with excellent resolution of his deficits. His anticoagulation was discontinued 1 week before admission in anticipation of dental extraction. Unfortunately, he then developed new left sided weakness involving his face, arm, and leg. General medical examination was unremarkable. On neurological examination, the only remarkable features were weakness in the proximal and distal muscles of the left arm and leg (2/5), and proximal and distal muscles of the right leg (4/5). Sensory examination was entirely within normal limits. Deep tendon reflexes and plantar flexor reflex were increased on the left. Routine laboratory evaluation, including electrolytes and hematocrit, was normal. Computed tomography (CT) demonstrated a single infarct in the right supplementary motor area and multiple deep infarcts in the right corona radiata. Single photon computed tomography (SPECT) confirmed decreased CBF in the right anterior cerebral artery-middle cerebral artery watershed. Duplex Doppler ultrasonography and magnetic resonance angiography of the carotid arteries revealed $80 \%$ stenosis in the left and occlusion in the right carotid arteries. After initially improving strength to $3 / 5$ proximally and $2 / 5$ distally in the left arm he experienced several additional episodes of transient worsening, so that by 1 week post-stroke his strength was $2 / 5$ proximally and $1 / 5$ distally. A repeat CT scan 8 days after admission showed extension of the right supplementary motor area infarct with hemorrhage, and absence of a flow void in the right carotid system, consistent with occlusion and low flow. Based on these findings, the patient was scheduled for left CEA in an attempt to prevent further contralateral flow failure.

\section{Anesthesia}

The patient was brought into the operating room. Routine monitoring (electrocardiography, pulse oximetry, and noninvasive blood pressure), radial arterial catheterization, transcranial Doppler ultrasonography (500 M, Multigon Industries Inc., Yonkers, NY), and EEG were performed. Preinduction medications included fentanyl $(50 \mu \mathrm{g})$ and midazolam $(2 \mathrm{mg})$. The patient was denitrogenated with $100 \%$ oxygen and induced with etomidate $(20 \mathrm{mg})$. Cisatracurium was used to facilitate intubation. An endotracheal tube was inserted uneventfully. Anesthesia was maintained with $\mathrm{N}_{2} \mathrm{O}$ in oxygen (70:30) and isoflurane ([0.5\% $]_{\text {exp }}$ ).

Phenylephrine (16.8 $\mathrm{mg}$ total dose) was administered as a continuous infusion to maintain the systolic blood pressure between 170 and $200 \mathrm{~mm} \mathrm{Hg}$ (prior to carotid artery cross-clamping) and between 200 and $220 \mathrm{~mm} \mathrm{Hg}$ (after carotid artery cross-clamping). Labetalol (30 $\mathrm{mg}$ ) was given after carotid artery cross-clamping to lower the systolic blood pressure to approximately $120 \mathrm{~mm} \mathrm{Hg}$.

\section{EEG}

Electroencephalography monitoring was used to determine the adequacy of CBF to maintain functional cerebral activity. The EEG was connected as an eight channel monopolar montage using an Electro-Cap (Electrocap International Inc., Eaton, $\mathrm{OH}$ ) with eight hemispheric electrodes $\left(\mathrm{F}_{3}, \mathrm{~F}_{4}, \mathrm{C}_{3}, \mathrm{C}_{4}, \mathrm{P}_{3}, \mathrm{P}_{4}, \mathrm{~T}_{5}\right.$ and $\left.\mathrm{T}_{6}\right)$ referred to a $\mathrm{C}_{\mathrm{Z}}$ reference. The $\mathrm{EEG}$ was recorded with a Neurotrac II (Moberg Medical, Ambler, PA) and stored on a removable hard drive (Syquest 270 MB, Syquest Inc., Fremont, CA) for later review. It was viewed in both raw and compressed spectral array format after processing in order to calculate spectral edge frequency. Spectral edge frequency was set at $97 \%$ of total EEG power. 


\section{Transcranial Doppler}

A $2 \mathrm{MHz}$ pulsed Doppler ultrasound probe (TCD) was applied to the right temporal area in order to determine the ipsilateral middle cerebral artery flow velocity. Determination of correct placement was made by measuring the velocity at different depths and seeing that the velocity remained relatively constant toward the probe, and at greater depth was both toward and away from the probe, consistent with the Doppler ultrasound being focused at the bifurcation of the middle cerebral artery and anterior cerebral arteries.

\section{Surgery}

The patient was placed supine with the head in an extended midline position tilted approximately $10^{\circ}$ to the right. An incision was made along a skin crease from just below the angle of the mandible to near the midline through skin, subcutaneous tissue, and platysma. The common, internal and external carotid arteries were exposed and controlled. During the course of the dissection there were dramatic changes in CBF velocity associated with pressing on the carotid artery. These alterations of CBF velocity were no longer apparent once manipulation of the carotid artery ceased (Fig. 1). After administering 5000 units of heparin intravenously, the common, internal and external carotid arteries were occluded. Cross-clamping of the left internal carotid artery resulted in very significant decreases in both CBF velocity and spectral edge frequency consistent with cerebral ischemia (Fig. 2). The EEG and TCD returned to baseline following insertion of a shunt (Fig. 3).

A longitudinal incision was made in the common carotid artery proximal to the bifurcation and extended into the internal carotid artery distal to the plaque. During the course of surgery, the shunt catheter was inadvertently kinked by the surgeon. The CBF velocity as measured by TCD decreased abruptly (Fig. 4). The change in CBF resolved immediately when the shunt was straightened. The atheroma was removed using a dissector. Firmly-attached intact intima was left above and below the area of atheroma resection. Before placing the final sutures, backbleeding from the common, internal, and external carotid arteries was performed and the lumen was washed with heparinized saline. Debris and air were expelled by releasing the clip on the superior thyroid artery, which provided inflow as the final sutures were secured. When the shunt was clamped for its removal, the EEG again demonstrated a decrease in SEF and the TCD showed a marked attenuation of CBF velocity (not shown). During removal of the shunt, the TCD tracings had high intensity transients and produced a chirping sound consistent with a shower of emboli (Fig. 5). Clamps were sequentially removed from the external, common, and internal carotid arteries. Once the carotid artery was reopened, both EEG and the CBF velocity returned to preoperative values (Fig. 6).

\section{Postoperative Course}

Upon completion of surgery the patient was awakened and extubated without incident. On his first postoperative neurological examination, he was weaker in his left arm (1/5 proximally and $0 / 5$ distally) and leg (2/5 proximally and 0/5 distally). He was then transferred to the neurological intensive care unit where emergent magnetic resonance angiography and carotid duplex showed a widely patent endarterectomy site without evidence of thrombus. A postoperative angiogram confirmed these findings and SPECT scan showed improved blood flow in the right hemisphere. Follow-up MRI and CT scan demonstrated further enlargement of the right anterior cerebral artery-middle cerebral artery watershed and supplementary motor area infarctions. Despite these radiographic findings the patient eventually made a dramatic recovery with the ability to walk independently. Nonetheless, his hand remained weak and he was unable to work as a truck driver. 


\section{Discussion}

\section{Indications for Transcranial Doppler Monitoring}

Transcranial Doppler Monitoring is frequently used to diagnose cerebral vascular disease, and to monitor $\mathrm{CBF}$ velocity and embolic rate during surgery on the carotid $(1,2)$. The intraoperative measurement of $\mathrm{CBF}$ velocity is valuable for two reasons. First, since changes in $\mathrm{CBF}$ velocity are proportional to changes in $\mathrm{CBF}$, TCD measurement serves as a surrogate monitor for changes in CBF (5). Second, changes in CBF velocity are seen in real time, without cumbersome equipment or complex and lengthy analysis.

\section{Determination of Cerebral Blood Flow Velocity}

There is considerable controversy whether TCD or EEG monitoring is more sensitive or specific for indicating cerebral areas at risk for ischemia. In our opinion, they complement each other. We report a case involving a patient undergoing CEA in whom TCD monitoring demonstrated impaired CBF at several points during surgery. During two of these episodes, the EEG tracings demonstrated no detectable changes. During the first episode, TCD monitoring clearly indicated that flow in the carotid artery was impaired during the initial dissection of the carotid artery. During the other episode, TCD monitoring revealed evidence of cerebral emboli not picked up by EEG. Additionally, TCD monitoring picked up all changes in $\mathrm{CBF}$ which became manifest on EEG, such as during cross-clamping and shunt kinking. These changes were immediately reported by the anesthesiologist to the surgeon, who was then able to correct the situation and promptly restore blood flow.

Continuous TCD monitoring of ipsilateral middle cerebral artery velocities provides beat-tobeat monitoring of the integrity of the shunt which EEG monitoring does not always reveal. Since this patient suffered an extension of his flow-related contralateral watershed CVA, the EEG-TCD mismatch was apparently clinically relevant. Although TCD also revealed changes during dissection which resulted in modification of surgical technique, it is less clear that these changes were clinically relevant.

\section{Acknowledgements}

The authors thank Multigon Industries, Inc., Yonkers, New York for lending us the TCD equipment. Dr. Heyer received partial salary support from the Charles A. Dana Foundation. Dr. Connolly is an Irving Assistant Professor of Neurological Surgery.

\section{References}

1. Halsey, JH, Jr. Monitoring blood flow velocity in the middle cerebral artery during carotid endarterectomy. In: Weschler, VbalR, editor. Transcranial Doppler, Clinical and Research Applications. New York: Mosby-Year Book Inc.; 1992. p. 216-21.

2. Halsey JH Jr. Risks and benefits of shunting in carotid endarterectomy. The International Transcranial Doppler Collaborators. Stroke 1992;23(11):1583-7. [PubMed: 1440706]

3. Berger, MP.; Tegeler, CH. Embolus detection using Doppler ultrasonography. In: Weschler, VbalR, editor. Transcranial Doppler, Clinical and Research Applications. New York: Mosby-Year Book Inc.; 1992. p. 232-41.

4. Gaunt ME, Martin PJ, Smith JL, et al. Clinical relevance of intraoperative embolization detected by transcranial Doppler ultrasonography during carotid endarterectomy: a prospective study of 100 patients. Br J Surg 1994;81:1435-9. [PubMed: 7820463]

5. Sorteberg W, Lindegaard KF, Rootwelt K, et al. Blood velocity and regional blood flow in defined cerebral artery systems. Acta Neurochir 1989;97:47-52. 


\section{TCD and EEG Before X-Clamp}

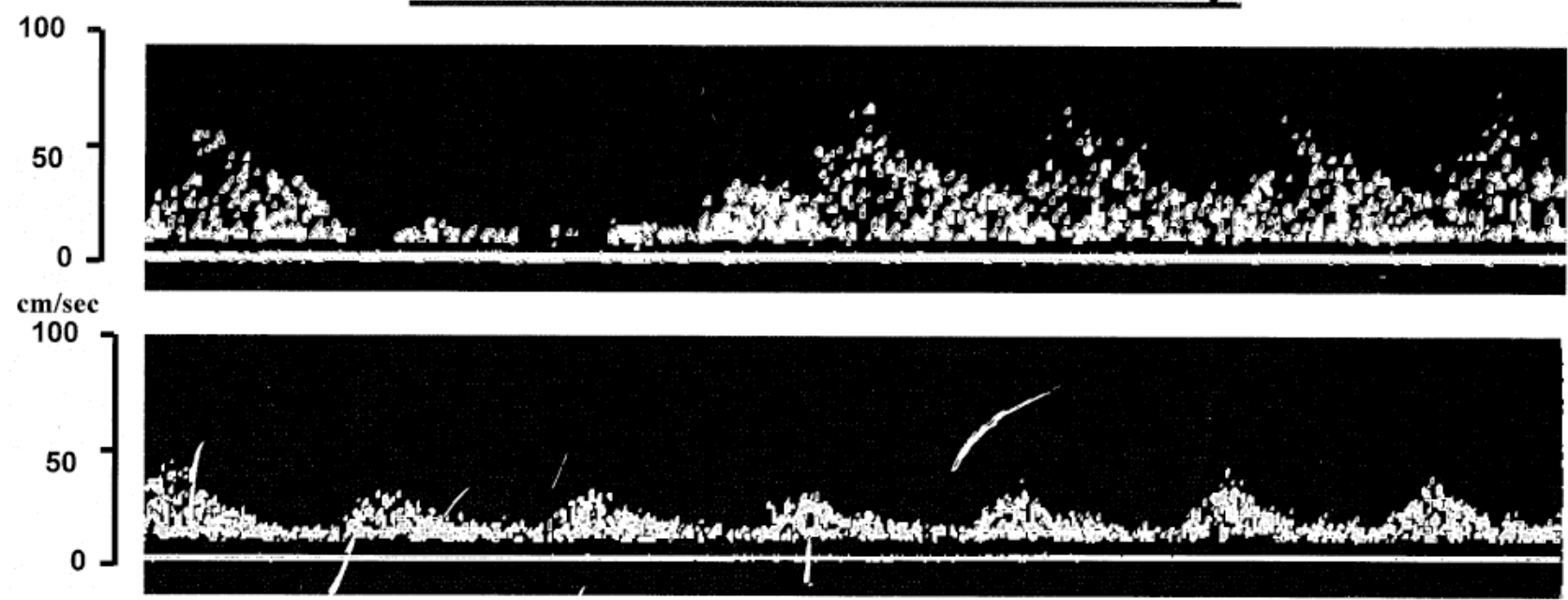

$\mathrm{cm} / \mathrm{sec}$

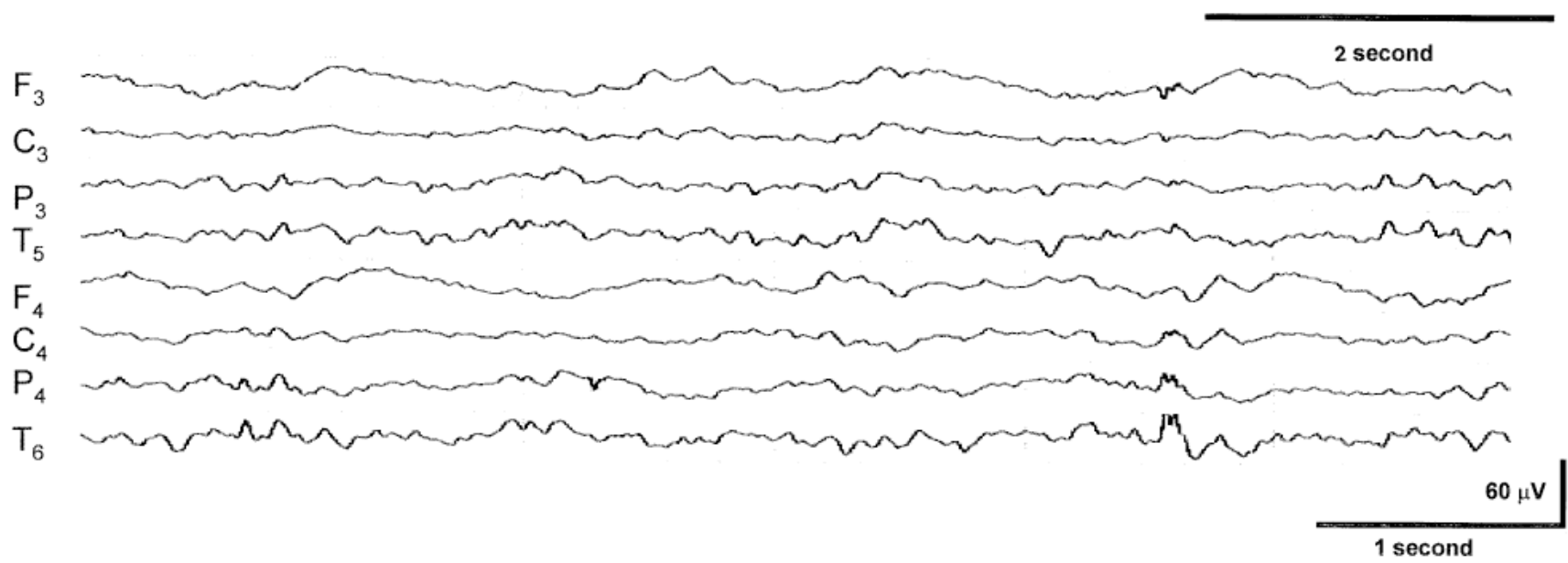

FIG. 1.

TCD and EEG tracings made prior to cross-clamping of the carotid artery. In the top strip, normal pulsatile flow is briefly interrupted by episodes of impaired flow caused by manipulation of the carotid artery by the surgeon. The second strip shows reduced blood flow velocity during an episode of sustained carotid artery manipulation. These transient changes in cerebral blood flow were not coincident with changes in EEG, seen in the bottom strip, which remained at baseline activity throughout this period of carotid artery manipulation. 


\section{TCD and EEG During X-Clamp}

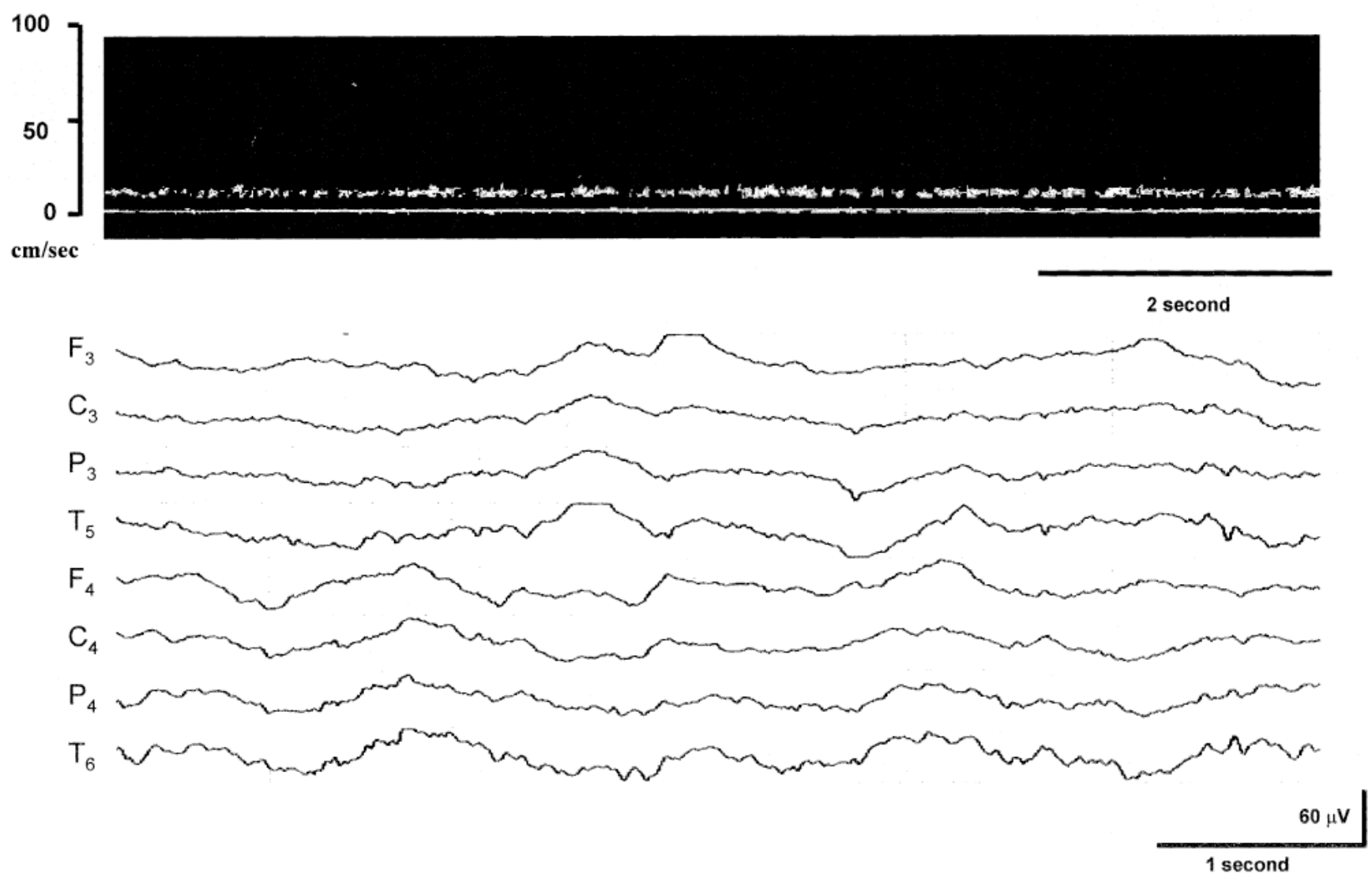

FIG. 2.

During cross-clamping of the carotid artery, both the TCD and the EEG showed dramatic changes, representing substantial flow failure in the cerebral vessels and attenuation of cerebral electrical activity. 


\section{$\underline{\text { TCD and EEG After Shunt Inserted }}$}
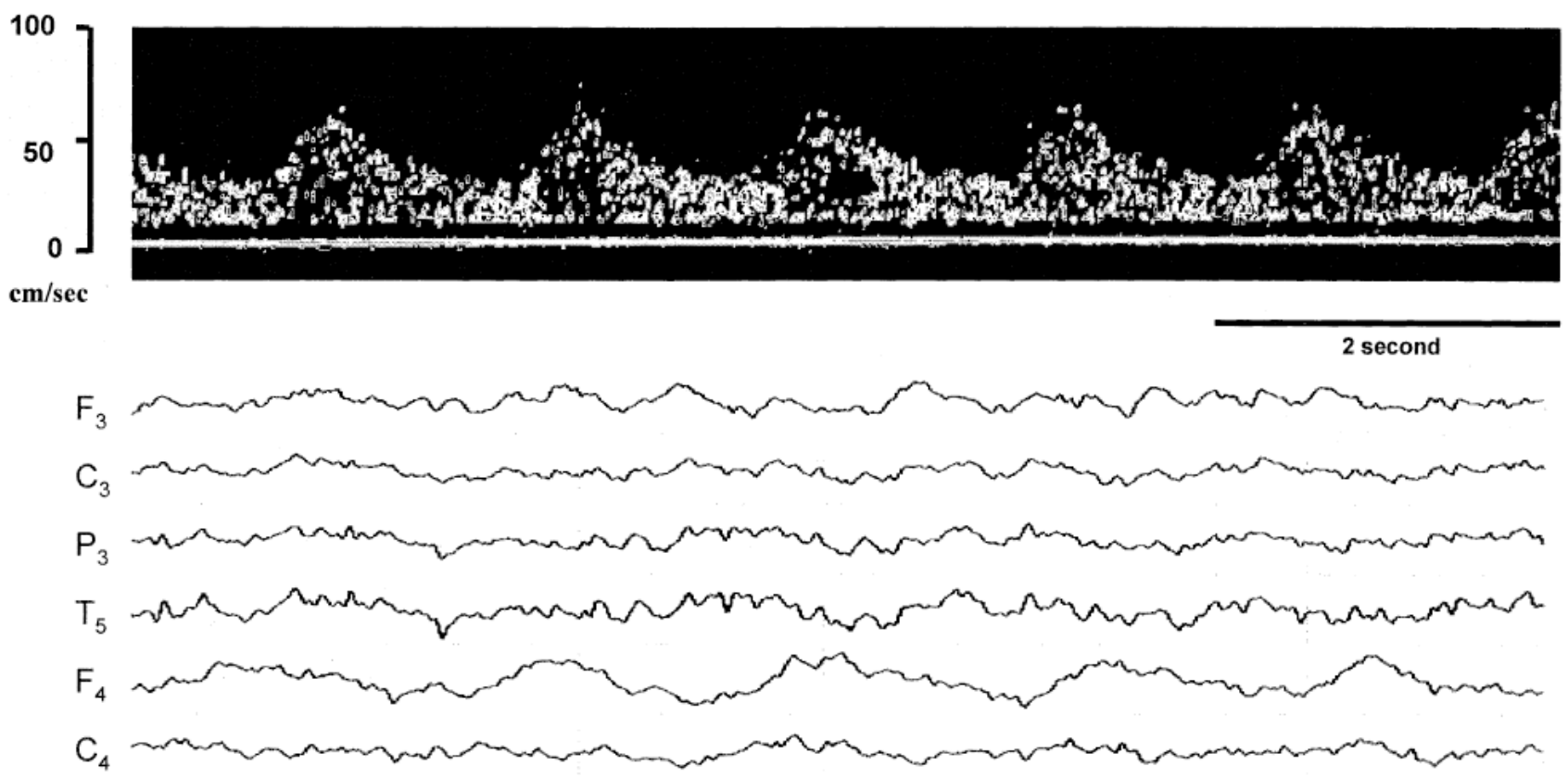

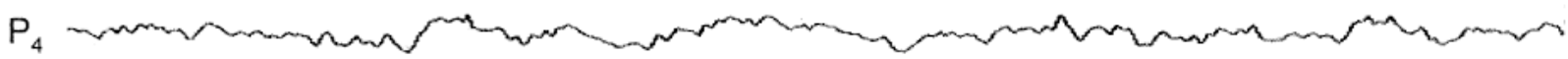

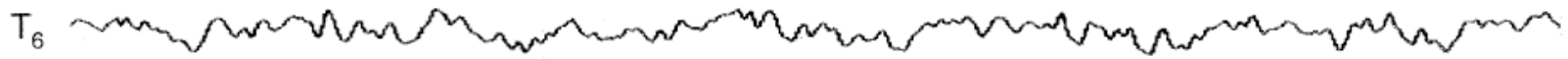

1 second

FIG. 3.

Following placement of a shunt, both the TCD and the EEG returned to baseline levels. 


\section{TCD and EEG While Shunt Present}

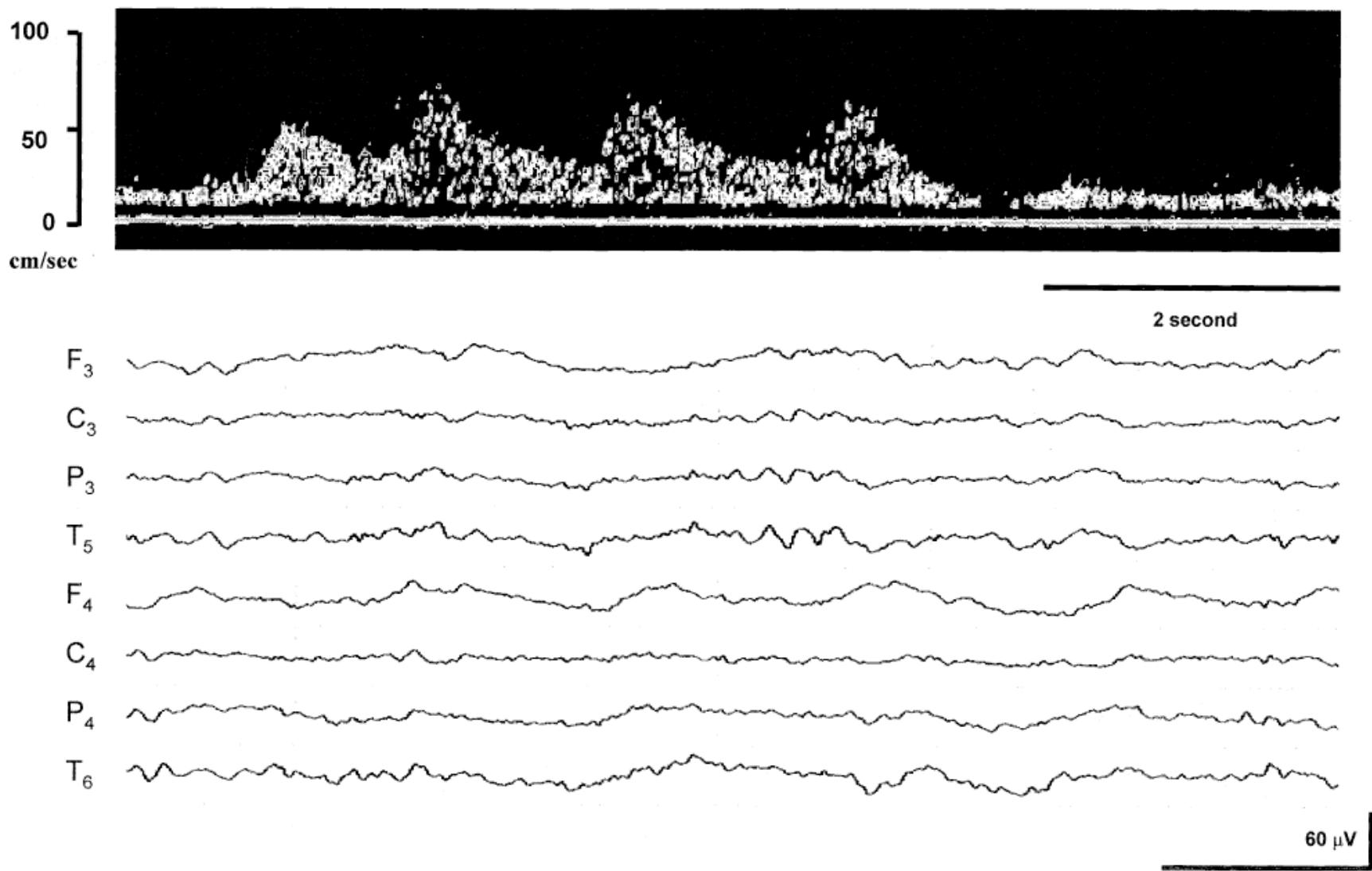

1 second

FIG. 4.

During cross-clamping the shunt was inadvertently kinked by the surgeon, causing a severe reduction in flow by TCD at the end of the top tracing. There were no changes in the EEG tracings during the transient interruption of blood flow through the shunt. 


\section{TCD At Shunt Removal}

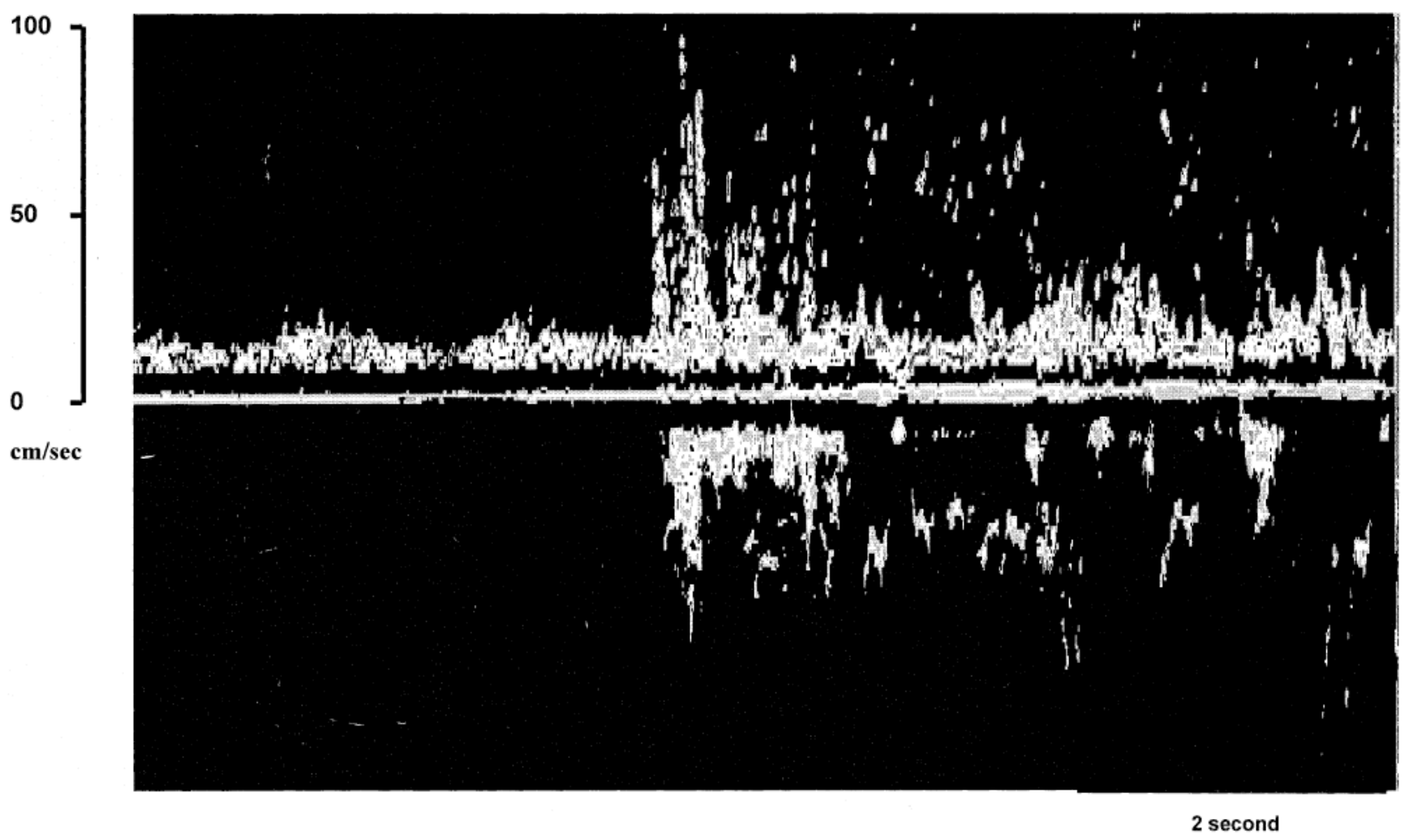

FIG. 5.

During removal of the shunt, the TCD demonstrated flow abnormalities consistent with a shower of emboli in the carotid system. At this same time, the EEG was resuming its gradual, uneventful return to baseline levels following resumption of carotid circulation. 


\section{TCD and EEG After Shunt Removed}
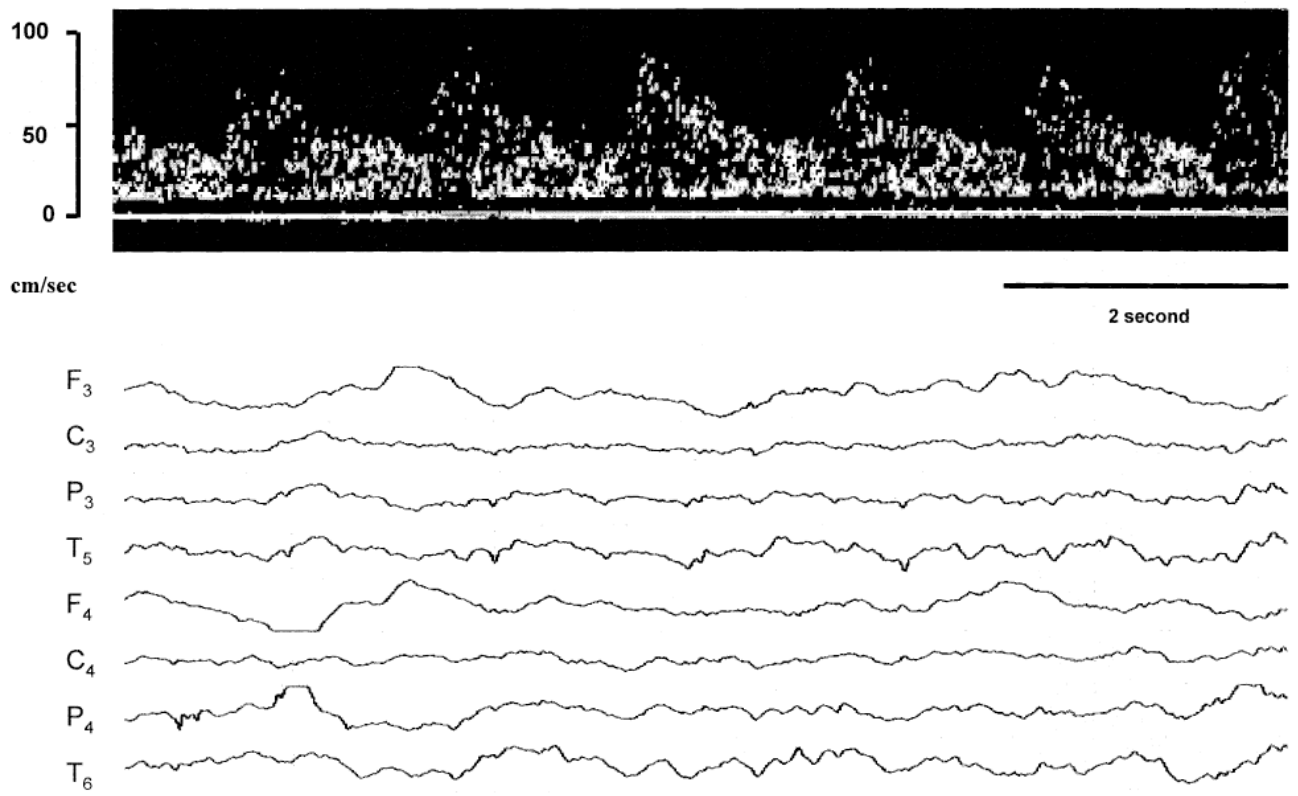

$60 \mu \mathrm{v}$

1 second

FIG. 6.

The TCD and EEG both returned to baseline levels several minutes after the removal of the shunt. 